\title{
LOS YACIMIENTOS PALEONTOLÓGICOS DE LA CUENCA TERCIARIA CONTINENTAL DE RUBIELOS DE MORA (ARAGÓN)
}

\author{
Plinio MONTOYA ${ }^{1}$, Enrique PEÑALVER ${ }^{1}$, Francisco J. RUIZ-SÁNCHEZ1, \\ Carlos de SANTISTEBAN ${ }^{1}$, Luis ALCALÁ2, Margarita BELINCHÓN ${ }^{3}$ y \\ José I. LACOMBA ${ }^{1}$
}

\footnotetext{
1 Departament de Geologia. Universitat de València. Dr. Moliner, 50, 46100 Burjassot.

${ }^{2}$ Museo Nacional de Ciencias Naturales, C.S.I.C., José Gutiérrez Abascal, 2, 28006 Madrid.

${ }^{3}$ Museu Paleontològic Municipal, Plaça de l'Ajuntament, 1, 46002 València.
}

\begin{abstract}
Montoya, P., Peñalver, E., Ruiz-Sánchez, F.J., Santisteban, C. de, Alcalá, L. Belinchón, M. y Lacomba, J.I. 1996. Los yacimientos paleontológicos de la cuenca terciaria continental de Rubielos de Mora (Aragón). IThe paleontological sites at the terciary continental basin of Rubielos de Mora (Aragon)]. Revista Española de Paleontología, $\mathbf{N}^{*}$ Extraordinario, $215-224$. ISSN 0213-6937.
\end{abstract}

\begin{abstract}
In this paper, the main results from the numerous geological and paleontological studies carried out in the Tertiary continental basin of Rubielos de Mora (Spain) throughout this century are compiled.

Until now, the paleontological knowledge of this basin was limited to its western sector (Rubielos de Mora 1, 2 and 3, and Cerro del Porpol). However, new sites, from the central and eastern parts of the basin, are being presently under study: Río Rubielos, Alto de Ballester 1 and 2, and Alto de la Venta. First results from these sites are presented here.

Two new localities are considered as the most important ones: Alto de Ballester 1 and Río Rubielos. The first one, consisting of sandy clays, yielded a rich micromammal association, which allow us to date the site as Lower Miocene (Ramblian, biozone A). Macrommamals are also present, being of special relevance the remains of a Moropus (Chalicotheriidae).

Río Rubielos is a Konservat-Lagerstätte site, formed by oil-shales, with a rich and diverse fossil flora and fauna. The fauna consists mainly of Amphibia, Crustacea, Arachnida and Insecta.
\end{abstract}

Keywords: Rubielos de Mora, Spain, Ramblian, Lower Miocene, Insecta, Mammalia, oil-shales.

\section{RESUMEN}

En el presente trabajo se exponen los resultados más significativos de los numerosos estudios geológicos y paleontológicos realizados a lo largo de este siglo en la cuenca terciaria de Rubielos de Mora.

El conocimiento sobre el contenido paleontológico de la cuenca se limitaba, hasta ahora, a unos pocos yacimientos localizados en su extremo occidental (Rubielos de Mora 1, 2 y 3, y Cerro del Porpol). Recientemente se ha iniciado el estudio de nuevos yacimientos situados en las partes central y oriental de la cuenca (Río Rubielos, Alto de Ballester 1 y 2 , y Alto de la Venta), presentándose aquí los resultados obtenidos hasta el momento.

Entre estas nuevas localidades destacan Alto de Ballester 1 y Río Rubielos. La primera de ellas, incluida en facies de arcillas arenosas, ha proporcionado una rica asociación de micromamíferos que permite situar Alto de Ballester 1 en el Mioceno inferior (biozona A del Rambliense). Además, se han obtenido también macromamíferos entre los que destaca el hallazgo de restos de un calicotérido, atribuido al género Moropus.

Río Rubielos es un yacimiento del tipo Konservat-Lagerstätte, formado por ritmitas bituminosas, con una rica y variada flora y fauna fósiles. Esta última se halla constituida básicamente por anfibios, crustáceos, arácnidos e insectos.

Palabras clave: Rubielos de Mora, España, Rambliense, Mioceno inferior, Insecta, Mammalia, ritmitas bituminosas.

\section{MARCO GEOLÓGICO}

La cuenca terciaria de Rubielos de Mora está situada en el sector sureste de la Cordillera Ibérica, en el dominio tectónico conocido como "Zona de Enlace". Durante el Mioceno inferior-medio esta cuenca estuvo configurada como una fosa tectónica de tipo semigraben (Anadón, 1983; Anadón et al. 1988a y 1988b), controlada por la formación de una cubeta sinclinal en el substrato mesozoico, y condicionada por fallas normales de orientación ENE-WSW (Guimerà, 1990).

Entre los depósitos que constituyen el relleno de esta cuenca se han diferenciado dos conjuntos discordantes. El conjunto inferior, datado como Mioceno inferior-medio (Moissenet y Gautier, 1971; Godoy y Anadón, 1986) aflora dentro de los límites marcados por las estructuras de fractura que han determinado la cubeta sedimentaria. El conjunto superior, atribuido al intervalo Mioceno superior-Plioceno por Moissenet y Gautier (1971), se extiende más allá de los límites tectónicos de la cuenca.

Anadón et al. (1988b) subdividen los depósitos de edad Mioceno inferior-medio en tres unidades (Unidad Inferior, Media y Superior), que afloran de manera solapante desde el margen norte hacia el margen sur. El espesor conjunto de las tres unidades totaliza entre 600 y $800 \mathrm{~m}$ (Anadón et al., 
1988a y 1988b). De todas las unidades estratigráficas reconocidas en la cuenca, sólamente la Unidad Superior contiene restos paleontológicos que permiten dar una atribución de edad, siendo todavía incierta la datación de los 200 a 400 m inferiores. Esta unidad tiene un espesor variable entre 0 y 400 $\mathrm{m}$ y aflora sólamente en la mitad meridional de la cuenca. Los mayores espesores de dicha Unidad aparecen en la zona próxima a la fractura que limita el margen sur de la cuenca. La Unidad Superior está formada por un sistema de varias asociaciones de facies, descritas por Anadón et al. (1988a), entre las que se incluyen depósitos fluviales, depósitos deltaicos, brechas gravitacionales, así como carbonatos y margas lacustres. En relación con las unidades y asociaciones de facies descritas por Anadón et al. (1988a), todos los yacimientos estudiados están emplazados en los depósitos de la Unidad Superior. Alto de Ballester 1 y Río Rubielos se hallan en la facies de limolitas laminadas $\left(\mathrm{C}_{1}\right.$ "laminated mudstones" de Anadón et al., 1988a) y Rubielos de Mora 1, Rubielos de Mora 2, Rubielos de Mora 3, Alto de la Venta y Cerro del Porpol en la facies de ciclos de limolitas laminadas y masivas $\left(\mathrm{C}_{3}\right.$ "laminated and massive mudstones cycles" de Anadón et al., 1988a).

Los primeros estudios de la cuenca se centran en los aspectos meramente geológicos, basados en su potencial minero. Fernández Navarro (1914) aporta unos primeros datos sobre la geología de la cuenca y calcula sus posibilidades petrolíferas. Gavala (1921) realiza ya un estudio geológico más completo y estima la capacidad de explotación de los lignitos y pizarras bituminosas, asignando a la cuenca una edad Oligoceno.

\section{Serie del Alto de Ballester}

En el presente trabajo se ha levantado una serie a lo largo de un pequeño barranco tributario del río Rubielos que drena el sector sur del Alto de Ballester (Fig. 1). En esta zona, situada a 1'5 Km al NE de Rubielos de Mora, afloran los materiales de la Unidad Superior bajo la forma de brechas gravitacionales y limolitas calcáreas laminadas conteniendo intercalaciones de black-shales. En esta serie se pueden diferenciar tres tramos desde la base hacia el techo (Fig. 2).

El tramo "A" está adosado, mediante un contacto mecánico, al substrato mesozoico. Está formado por $45 \mathrm{~m}$ de bre-

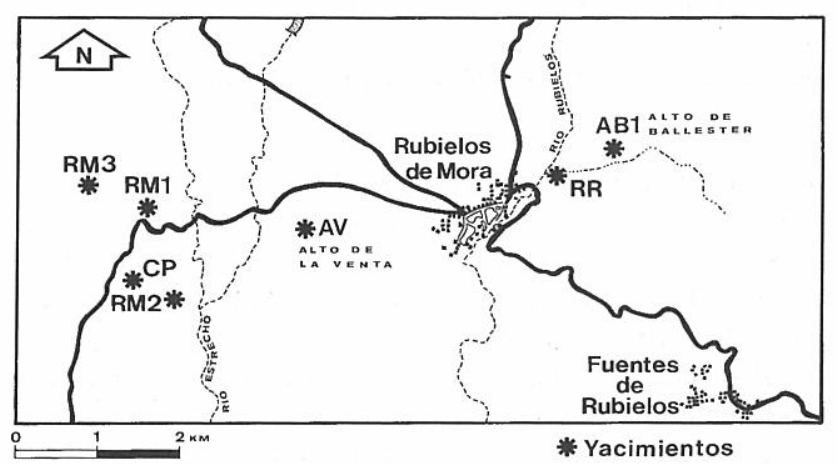

Figura 1. Situación geográfica de los yacimientos de Rubielos de Mora referidos en este trabajo. AB1: Alto de Ballester 1; AV: Alto de la Venta; CP: Cerro del Porpol; RMI, RM2, RM3: Rubielos de Mora 1, 2 y 3; RR: Río Rubielos.

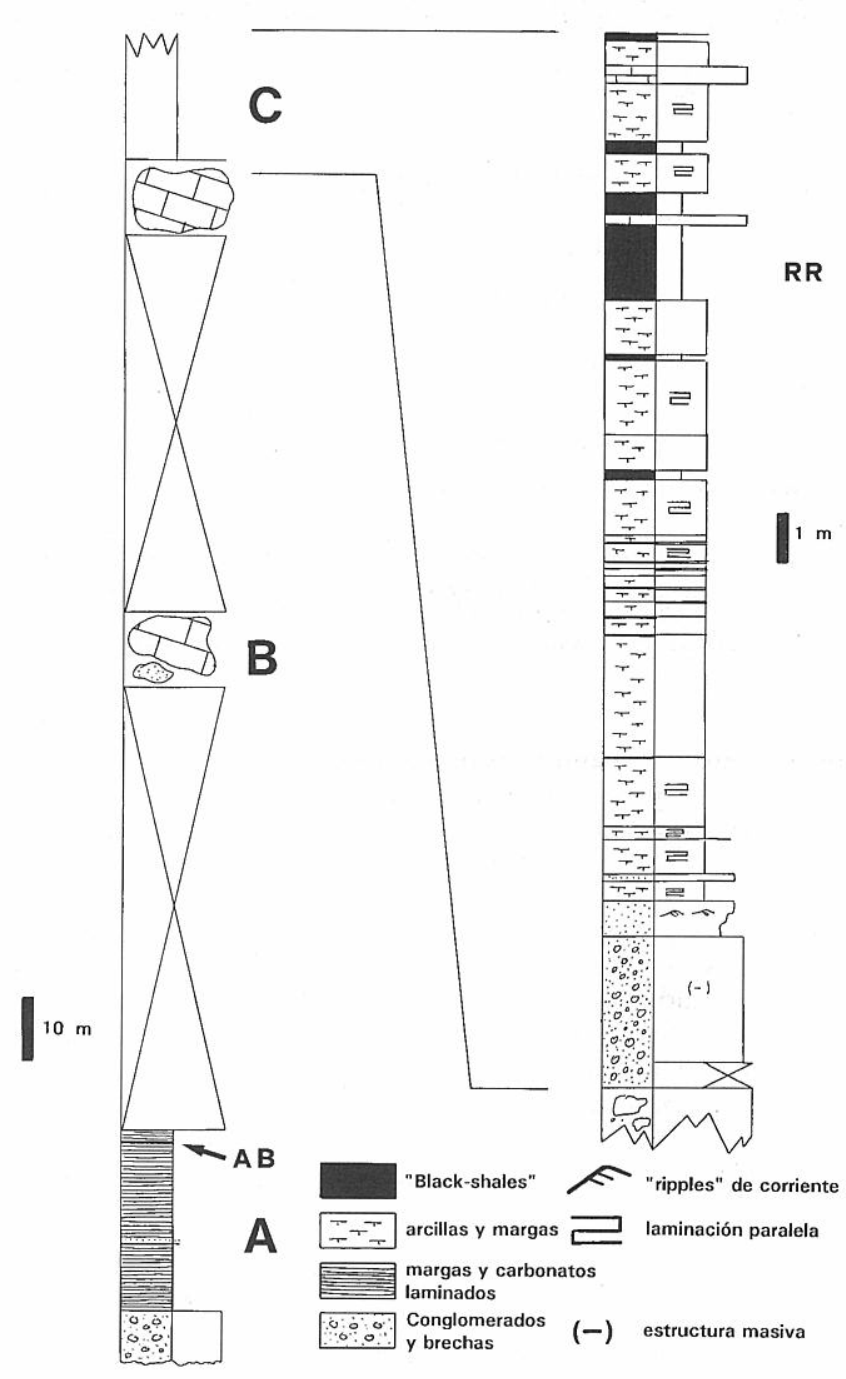

Figura 2. Columna estratigráfica con localización de los yacimientos Alto de Ballester (AB) y Río Rubielos (RR).

chas y conglomerados intercalados con areniscas y arcillas arenosas grises. Las brechas, que pueden ser consideradas como coluviones formados al pie de un escarpe de falla, predominan en la parte inferior, mientras que hacia el techo son más abundantes las arcillas y areniscas. Estas últimas presentan grietas de retracción por desecación. En la parte alta de este tramo se halla el yacimiento de Alto de Ballester 1.

El tramo "B" está formado por $80 \mathrm{~m}$ de arcillas grises y ocres que incluyen dos niveles continuos de olistolitos de carbonatos y areniscas del Cretácico, los cuales pasan, hacia el SW, a un olistostroma de $4 \mathrm{~m}$ de espesor formado por una masa caótica de areniscas pobremente cementadas y cantos. En el techo de este olistostroma existe un nivel con ripples de corriente que marcan un sentido de desarrollo del flujo sedimentario hacia el NNW.

El tramo "C" se halla dispuesto de forma neta sobre los materiales del tramo "B". Está formado por $16 \mathrm{~m}$ de limolitas calcáreas laminadas que incluyen intercalaciones de 1 $\mathrm{mm}$ a 1 ' $5 \mathrm{~m}$ de espesor de black-shales, y esporádicamente algún nivel de calizas estromatolíticas. En este tramo se engloba el yacimiento del Río Rubielos. 


\section{ANTECEDENTES PALEONTOLOGICOS}

La primera cita acerca de los fósiles de la cuenca se debe a Hernández-Sampelayo y Cincúnegui (1926), los cuales mencionan restos vegetales (hojas y semillas) que asemejan a los hallados en la cuenca de Ribesalbes-Alcora (Castellón). Estos autores citan la presencia, en la cuenca, de los géneros Salix (Salicaceae), Corylus y Ostrya (Betulaceae).

El grueso de los trabajos paleontológicos comienza a partir de mediada la década de los años 60 con el trabajo de Crusafont et al. (1966). Estos autores dan a conocer la existencia del primer yacimiento de vertebrados encontrado en la cuenca. Bruijn y Moltzer (1974) sitúan esta localidad, a la que denominan Rubielos de Mora 1 (RM1), en unas capas de arenas que constituyen la parte superior del relleno lacustre de la cuenca en su sector centro-occidental. López-Martínez (1989) estudia los restos de lagomorfos proporcionados por este yacimiento. Así, la lista faunística de Rubielos de Mora 1, a partir de estos trabajos, además de datos propios, quedaría configurada según se refleja en la Tabla 1.

Aguirre y Moissenet (1972) descubren un nuevo yacimiento del que se recuperaron restos de un rinoceronte del género Brachypotherium, localidad que denominaremos Rubielos de Mora 3 (RM3). Ésta se sitúa en niveles arcilloarenosos pertenecientes al conjunto inferior (Mioceno inferior-medio) del relleno de la cuenca. La posterior asignación de dichos restos a la especie Brachipotherium aurelianense, por parte de Cerdeño (1989), indicaría una edad RamblienseAragoniense inferior para el yacimiento.

\begin{tabular}{|c|c|c|c|c|c|}
\hline & & & \\
\hline & & & RMI & RM2 & $\mathrm{AB} 1$ \\
\hline \multirow[t]{5}{*}{ Insectivora } & Erinaceidae & Galerix sp. & 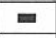 & & \\
\hline & Soricidae & Sorex sp. & $=$ & & \\
\hline & \multirow[t]{2}{*}{ Talpidae } & Talpidac indet. & $=$ & & \\
\hline & & Desmanella fejfari & & $\square$ & \\
\hline & Dimylidae & Cordylodon intercedens & & $=$ & \\
\hline \multirow[t]{3}{*}{ Lagomorpha } & \multirow[t]{3}{*}{ Ochotonidae } & Prolagus vasconiensis & $=$ & & $=$ \\
\hline & & Lagopsis peñai & 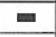 & & $=$ \\
\hline & & Lagopsis cf. peñai & & $=$ & \\
\hline \multirow[t]{12}{*}{ Rodentia } & \multirow[t]{6}{*}{ Gliridac } & $P_{\text {seudodryomys simplicidens }}$ & & $=$ & \\
\hline & & Pseudodryomys aff. ibericus & $=$ & $=$ & $=$ \\
\hline & & Myoglis sp. & & $=$ & \\
\hline & & Glirudinus modestus & & $=$ & \\
\hline & & Peridyromys aquatilis & & $=$ & 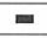 \\
\hline & & Peridyromys aff. murinus & & & = \\
\hline & \multirow[t]{3}{*}{ Eomyidae } & Pseudotheridomys feifari & & $=$ & $=$ \\
\hline & & Pseudotheridomys sp. & $=$ & & $=$ \\
\hline & & Ligerimys sp. & & $=$ & \\
\hline & Sciuridae & Blackia miocaenica & & $=$ & \\
\hline & Cricetidae & Neocometes similis & & $=$ & \\
\hline & \begin{tabular}{|l|} 
Castoridae \\
\end{tabular} & Castoridae indet. & & & 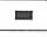 \\
\hline Carnivora & Mustelidae & Mustelidae indet. & & & 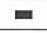 \\
\hline \multirow[t]{7}{*}{ Perissodactyla } & \multirow[t]{2}{*}{ Equidae } & Anchitherium aurelianense & $=$ & & \\
\hline & & Anchitherium sp. & & $=$ & $=$ \\
\hline & \multirow[t]{3}{*}{ Rhinocerotidae } & Dicerorhinus cf. tagicus & $=$ & & \\
\hline & & Dicerorhinus sp. & 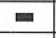 & & \\
\hline & & cf. Brachypotherium sp. & & & $=$ \\
\hline & \multirow[t]{2}{*}{ Chalicotheriidae } & Chalicotheriidae indet. & $=$ & & \\
\hline & & Moropus sp. & & & = \\
\hline \multirow[t]{3}{*}{ Artiodactyla } & \multirow[t]{2}{*}{ Palaeomericidae } & Palaeomeryx kaupi & $=$ & & \\
\hline & & cf. Palaeomeryx sp. & & & $=$ \\
\hline & Cervidae & Procervulus dichotomus & $=$ & & $=$ \\
\hline
\end{tabular}

Tabla 1. Listas faunísticas de los mamíferos de Rubielos de Mora 1 (RM1), Rubielos de Mora 2 (RM2) y Alto de Ballester 1 (AB1).
Bruijn y Moltzer (1974) inician el estudio de la fauna de microvertebrados en una nueva localidad que denominan Rubielos de Mora 2 (RM2), la cual se engloba en una capa de arcillas arenosas verdes, muy rica en gasterópodos, que aflora en el barranco que discurre por la ladera sur del Cerro del Porpol. Estos autores asignan al yacimiento una edad Mioceno inferior y unas condiciones ecológicas de alta humedad con amplia forestación. Gibert (1974 y 1975) estudia los insectívoros y define en esta localidad la nueva especie Desmanella fejfari. Por su parte, Sanchiz (1977) cita en el mismo yacimiento los anfibios Chelotriton paradoxus y Rana pueyoi. Por último, Álvarez Sierra y Daams (1987) revisan los Eomyidae. De este modo, la lista de los mamíferos de Rubielos de Mora 2, con la aportación de datos propios, queda establecida según se observa en la tabla 1.

La década de los años 80 es prolija en trabajos científicos en el área de Rubielos de Mora. Así, Anadón (1983), Anadón et al. (1988b, 1989 y 1990), Moissenet (1989), Simón (1984), y Prado et al. (1988) aportan nuevos datos geológicos de la cuenca. Por otra parte, en esta misma década Cerdeño (1989) estudia los restos de rinocerótidos, determinando específicamente los restos del rinoceronte de Rubielos de Mora 3 como Brachypotherium aurelianense. Los restos de lagomorfos de los yacimientos Rubielos de Mora 1 y 2 son estudiados por López-Martínez (1977 y 1989) atribuyendo a Rubielos de Mora 2 una edad Aragoniense inferior; según esta autora RM2 es más reciente que la localidad valenciana de Buñol, atribuida a la zona MN 4 (Mein, 1990; Bruijn et al., 1992).

Reyes y Feixas (1984) analizan y tipifican las pizarras bituminosas señalando la presencia, en la fase orgánica, de microalgas entre las que se incluye Botriococcus. Por otro lado, Fernández-Marrón y Álvarez-Ramis (1988) realizan un estudio previo sobre la flora de la cuenca, y ÁlvarezRamis y Fernández-Marrón (1994) llevan a cabo un estudio paleobotánico en el que relacionan los palinomorfos con los macrorestos. Las muestras, al parecer del yacimiento de Rubielos de Mora 2 (Fernández-Marrón, com. pers.), han permitido reconocer en la cuenca las familias Pinaceae, Taxodiaceae, Myricaceae, Betulaceae, Fagaceae, Salicaceae, Ulmaceae, Juglandaceae, Aceraceae, Potamogetonaceae, Typhaceae, Sparganaceae, Poaceae y Dictiosferiaceae, entre otras.

Bruijn y Moltzer (1974) mencionan por vez primera la presencia de insectos fósiles en la cuenca, en unos niveles de lutitas laminadas que citan como "RM Insecta II", aunque no llevan a cabo ninguna determinación sistemática. Estos afloramientos se sitúan en la ladera sur del Cerro del Porpol, hacia el techo de la serie que aflora en el mismo barranco donde se encuentra Rubielos de Mora 2.

En esta misma área hemos prospectado un nivel de ritmitas bituminosas con insectos y flora, cuya posición coincide con la del yacimiento de Bruijn y Moltzer, al que hemos denominado Cerro del Porpol (CP). Se han obtenido en la prospección ejemplares de insectos pertenecientes a los órdenes Odonata, Orthoptera, Homoptera, Heteroptera, Thysanoptera (lám. 2, fig. 13), Coleoptera (lám. 2, fig. 15), Hymenoptera (lám. 2, fig. 14) y Diptera. Entre los fósiles obtenidos podemos destacar dos interesantes ejemplares de homópteros de la familia Psyllidae (lám. 2, fig. 12) y un heteróptero de la familia Tingidae. La abundancia de insectos fósiles y su esta- 
do de conservación son similares a las existentes en el yacimiento del Río Rubielos.

\section{NUEVOS YACIMIENTOS}

\section{Alto de Ballester 1 (AB1)}

Este yacimiento, que fue descubierto a principios de 1990, se halla situado a unos $1500 \mathrm{~m}$ al ENE de la población de Rubielos de Mora, en el sector sur del paraje conocido como Alto de Ballester. Se localiza en la parte alta del tramo "A" de la serie que hemos realizado (fig. 2). Las capas fosilíferas consisten en margas arenosas con vertebrados y limos laminados con vegetales, y afloran en un pequeño corte situado en una zona de terreno abancalado, junto a una casa de labor.

En el verano de 1992 se llevó a cabo en este yacimiento una campaña de excavación en la que se obtuvo una amplia colección de micromamíferos, aunque desgraciadamente el yacimiento se mostró sumamente pobre en restos de macromamíferos. De todos modos, el hecho que más destaca en Alto de Ballester 1 es el hallazgo de restos óseos de un calicotérido (Perissodactyla, Chalicotheriidae), grupo éste francamente escaso en el registro fósil.

Los fósiles obtenidos en este yacimiento se hallan depositados en los siguientes centros: Museo Paleontológico de la Universidad de Zaragoza (MPZ), Museo Paleontológico Municipal de Valencia (MPV) y Departamento de Geología de la Universidad de Valencia (DGV). También se refieren en el presente trabajo algunos ejemplares que obran en poder de D. Federico Alegre (CFA), vecino de Rubielos de Mora y dueño del terreno en que se encuentra el yacimiento de Alto de Ballester 1.

La lista de mamíferos, a falta de la determinación de los insectívoros, queda establecida tal como se presenta en la tabla 1.

Además de los mamíferos, son frecuentes en el yacimiento los restos óseos de anfibios, reptiles y aves, entre los que se ha podido determinar un coracoides de una anátida (A. Sánchez, com. pers.). También hay abundantes gasterópodos (helícidos) así como girogonitos de caráceas.

Por otra parte, en la capa de limos laminados se han encontrado restos esqueléticos articulados del anfibio Chelotriton, junto a una asociación de vegetales compuesta por grandes concentraciones de semillas del género Potamogeton, además de diferentes tipos de hojas. También aparecen en estas facies huevos durables de crustáceos cladóceros del género Daphnia, así como un gran número de ostrácodos.

Los restos de micromamíferos encontrados en Alto de Ballester 1 pertenecen en gran parte al orden Rodentia. Entre ellos, son más numerosos los pertenecientes a la familia Gliridae, representada por los géneros Peridyromys y Pseudodryomys (Fig. 3). En menor cuantía se halla representada la familia Eomyidae con el género Pseudotheridomys (Fig. 4), que es también muy significativo desde el punto de vista bioestratigráfico. La presencia exclusiva de restos de glíridos y eomíidos nos lleva a incluir a Alto de Ballester 1 en el "cricetid vacuum", al igual que otros yacimientos de la Península Ibérica, tales como Moratilla, Agreda, Bañón y La Dehesa (Daams y Freudenthal, 1990).
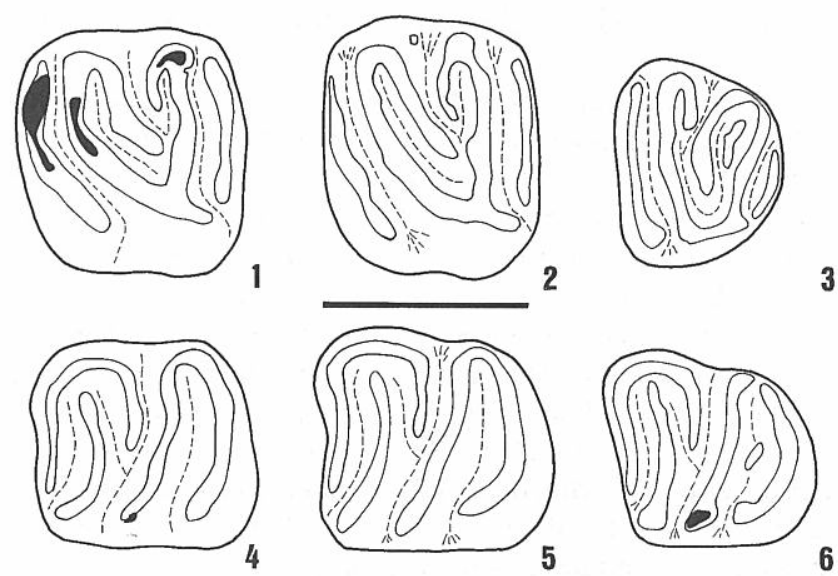

Figura 3. Dentición de Pseudodryomys aff. ibericus de Alto de Ballester 1. DGV: Departamento de Geología de la Universidad de Valencia. Escala $=1 \mathrm{~mm} .1: \mathrm{M}^{1}$ izquierdo (DGV Ab-22). 2: $\mathrm{M}^{2}$ izquierdo (DGV AB-48). 3: $\mathrm{M}^{3}$ izquierdo (DGV AB-67). 4: $\mathrm{M}_{1}$ izquierdo (DGV AB-8). 5: $M_{2}$ izquierdo (DGV AB-29). 6: $\mathrm{M}_{3}$ izquierdo (DGV AB-64).

Estos mismos autores argumentan que el Aragoniense comienza con la aparición de cricétidos modernos, como Democricetodon. La presencia de Pseudotheridomys feifari, junto a la inexistencia de restos de cricétidos modernos indica para Alto de Ballester 1 una edad anterior al Aragoniense, dentro de la biozona A del Rambliense, según la biozonación de Daams et al. (1987).

La composición de las listas faunísticas de Alto de Ballester 1 y de Rubielos de Mora 2 presentan una gran semejanza. La diferencia más aparente es la presencia en la segunda de estas localidades del cricétido Neocometes similis que, por otra parte, no es significativo desde el punto de vista biocronológico. Por tanto, podemos suponer para ambas localidades una edad similar.
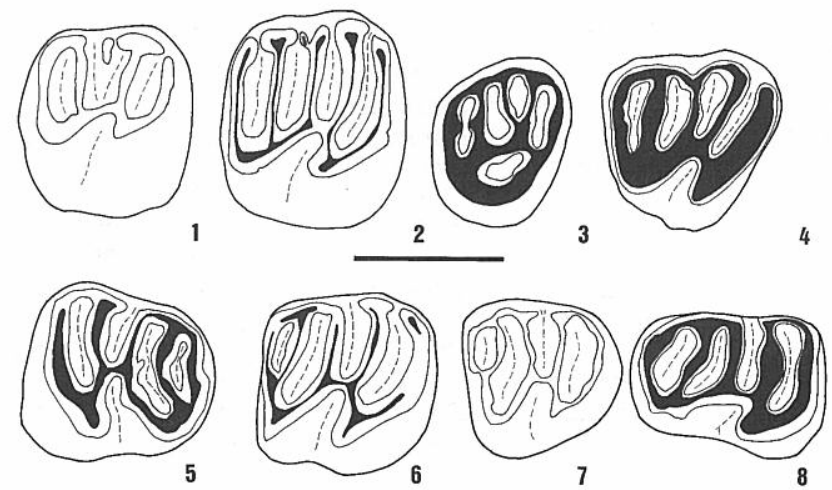

Figura 4. Dentición de Pseudotheridomys fejfari Álvarez-Sierra \& Daams, 1987 de Alto de Ballester 1. DGV: Departamento de Geología de la Universidad de Valencia. Escala $=1$ mm. 1: $\mathrm{P}^{4}$ derecho (DGV Ab-119). 2: $\mathrm{M}^{1-2}$ derecho (DGV AB-101). 3: $\mathrm{M}^{3}$ derecho (DGV AB-110). 4: $\mathrm{D}^{4}$ derecho (DGV AB-98). 5: $\mathrm{P}_{4}$ derecho (DGV AB-115). 6: $\mathrm{M}_{1-2}$ izquierdo (DGB AB-77). 7: $\mathrm{M}_{3}$ izquierdo (DGV $\mathrm{AB}-$ 105). 8: $\mathrm{D}_{4}$ izquierdo (DGV AB-123). 
Entre los macromamíferos, el grupo más abundante en el yacimiento es el de los ciervos (Lám. I, Figs. 1 y 2). Éste se encuentra representado por dientes, huesos y fragmentos de asta, que se ajustan muy bien a las dimensiones dadas para Procervulus dichotomus por Ginsburg y Bulot (1987), Antunes et al. (1994) y Azanza et al. (1993), mientras que quedarían cerca de las máximas de $P$. ginsburgi (Azanza, 1993; Azanza et al., op cit.).

Del género Anchitherium se han encontrado varios elementos dentarios (Lám. I, Figs. 3 y 4) y esqueléticos que, biométricamente, se sitúan por debajo de las tallas mínimas del A. aurelianense de Puente de Vallecas (Herráez y Alberdi, 1983) y de la forma de Córcoles (Iñigo, 1993), y en cambio se corresponden bien con la forma de pequeña talla que aparece en Buñol (Belinchón, 1987).

El calicotérido, perteneciente a la subfamilia Schizotheriinae, se halla representado por una asociación de restos óseos que corresponden a una misma extremidad posterior (Lám. I, Figs. 6-9). Se trata claramente de una forma de mayor talla que Phyllotillon naricus de Buñol (Belinchón y Montoya, 1989-1990). Los metatarsianos hallados en Alto de Ballester 1 muestran los caracteres diagnósticos para estos huesos, establecidos por Coombs (1978 y 1989) en el género Moropus: longitud subigual entre los metatarsianos III y IV, y presencia en éste último de una faceta para el ectocuneiforme.

A unos $300 \mathrm{~m}$, hacia el NE de la localidad Alto de Ballester 1 , se ha encontrado un afloramiento de lutitas grises, con abundantes restos de gasterópodos en superficie, al que hemos denominado Alto de Ballester 2 (AB2). El lavado de una muestra de prueba ha proporcionado una veintena de restos de micromamíferos fósiles. En este muestreo se encuentran representados los órdenes Rodentia, Insectivora y Chiroptera. Hasta el momento no se ha llevado a cabo la determinación específica de este material. No obstante, los primeros análisis nos hacen suponer una fuerte similitud con el material de micromamíferos encontrado en la localidad Alto de Ballester 1.

\section{Río Rubielos (RR)}

Este yacimiento, que fue dado a conocer por MartínezDelclòs et al. (1989), se localiza al sur del paraje de Alto de Ballester, a $500 \mathrm{~m}$ al NE de la población. Está constituido por ritmitas bituminosas que presentan varios afloramientos a lo largo de un pequeño barranco tributario del río Rubielos, y se engloba en el tramo "C" de la serie que hemos realizado (Fig. 2). Martínez-Delclòs et al. (1991) estudiaron el importante registro fósil de insectos, identificando 26 familias pertenecientes a 9 órdenes. Asimismo, citaron la presencia de otros artrópodos (Araneae y Ostracoda) y plumas de ave (Lám. II, Fig. 2). Por otra parte, Chica y Peñalver (1990) refieren un gran número de estados juveniles de anfibios urodelos en perfecta articulación y con impresión de los tejidos blandos (Lám. II, Fig. 3). También son comunes los ejemplares de hojas (Lám. II, Fig.1), flores, semillas y frutos.

Posteriormente a este primer estudio fue localizado, en la colección particular del Sr. Federico Górriz, un ejemplar de miriápodo de la familia Scutigerellidae, grupo muy escaso en el registro fósil. Aparte de los ostrácodos existe un abundante registro de huevos de resistencia (efipios) del crustáceo cladócero Daphnia.
Actualmente, el grupo de los insectos cuenta en el yacimiento al menos con 35 familias emplazadas en 12 órdenes. Los odonatos presentan un escaso registro de larvas y ningún adulto, hecho raro para este tipo de yacimientos. El orden Dermaptera, al igual que Raphidioptera, cuenta con un solo ejemplar hallado. Los saltamontes se encuentran en gran número y todos los ejemplares corresponden a una misma forma perteneciente a la familia Acrididae (Lám. II, Fig. 4); la presencia de gran número de ejemplares en algunos niveles puede ser indicativo de migraciones masivas típicas de esta familia. Entre los Homoptera destacan los pulgones, casi todos ellos pertenecientes a formas aladas (Lám. II, Fig. 8), hecho que corresponde a una mayor probabilidad de quedar atrapados en el agua del lago durante los desplazamientos en vuelo y por arrastre del viento. Del orden Heteroptera son comunes las formas terrestres (Lám. II, Fig. 7), habiéndose hallado dos ejemplares raros en el registro fósil; un ejemplar de la familia Stenocephalidae (MartínezDelclòs et al., 1991) y otro ejemplar de la familia Tingidae. Algunos coleópteros conservan parte de su volumen original debido a que este grupo suele tener la cutícula muy reforzada por una mayor esclerotización; son muy comunes en el yacimiento los ejemplares de Curculionidae y Staphylinidae (Lám. 2, Fig. 5). Los insectos fósiles del orden Hymenoptera presentan formas predadoras, nectívoras (Lám. II, Fig. 6) y especialmente parásitas. Los dípteros presentan un gran número de formas distintas (Lám. II, Figs. 9 y 10); son realmente abundantes los ejemplares de mosquitos de la familia Chironomidae, estando representados tanto machos como hembras. Este hecho es indicativo, para ciertas láminas en las ritmitas bituminosas, de periodos reproductivos con formación de enjambres.

El registro de formas acuáticas se compone principalmente de estados larvales y pupales de los ordenes Trichoptera y Diptera, así como de adultos y fases inmaduras de Heteroptera, como por ejemplo los Corixidae. Los tricópteros están presentes en forma de estuches larvales o carcajes, de muy variada morfología y composición, pero también en forma de estados pupales y adultos.

El yacimiento de Río Rubielos es localidad tipo para el lepidóptero Zygaena? turolensis, la especie más antigua de la familia Zygaenidae (Fernández-Rubio et al., 1991; Fernández-Rubio y Peñalver, 1994); de esta familia sólo se conocían otras dos especies del Mioceno alemán (Nauman, 1987).

Las características de la conservación de estos organismos han sido expuestas por Peñalver y Martínez-Delclòs (1993) y corresponderían a un yacimiento del tipo Konservat-Lagerstätte, caracterizado por un bajo grado de descomposición, lo cual se traduce en una baja tasa de desarticulación, en la preservación de estructuras delicadas (omatidios, pilosidad, escamas, etc.) y órganos internos (tracto digestivo y espermatecas), así como de patrones de coloración alar (Lám. II, Fig. 9), y una conservación diferencial según el grado de esclerotización.

Los restos fósiles de este yacimiento referidos en la presente publicación se hallan depositados en el Museo Paleontológico Municipal de Valencia (MPV). Los ejemplares obtenidos en la campaña de 1994, actualmente en estudio, están depositados en el Museo Paleontológico de la Universidad de Zaragoza (MPZ).

La lista faunística de los insectos de Río Rubielos se expone en el cuadro 2 , en el cual los taxones que se presentan en negrita son primeras citas para la cuenca. 


\begin{tabular}{|c|c|c|c|}
\hline & & Río Rubielos (RR) & $\begin{array}{c}\text { Alto de la } \\
\text { Venta (AV) }\end{array}$ \\
\hline \multirow[t]{2}{*}{ Odonata } & Libellulidae & $=$ & \\
\hline & Aeshnidae & $=$ & \\
\hline Dermaptera & Forficulidae? & $=$ & \\
\hline Orthoptera & Acrididae & $\square$ & $=$ \\
\hline \multirow[t]{4}{*}{ Homoptera } & Cercopidae & $=$ & \\
\hline & Aphididae & Aphidinae & 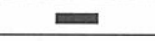 \\
\hline & Phylloxeridae & $=$ & \\
\hline & Psyllidae & Psylla sp., Trioza sp. & \\
\hline \multirow[t]{9}{*}{ Heteroptera } & Miridae & $=$ & \\
\hline & Reduviidae & $=$ & \\
\hline & Stenocephalidae & Dicranocephalus? sp. & \\
\hline & Coreidae & $=$ & \\
\hline & Pentatomidae & $=$ & \\
\hline & Notonectidae & $=$ & \\
\hline & Corixidae & Corixinae & Corixinae \\
\hline & Lygaeidae & $=$ & \\
\hline & Tingidae & $=$ & \\
\hline \multirow[t]{2}{*}{ Thysanoptera } & Thripidae & 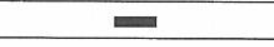 & $=$ \\
\hline & Aeolothripidae & $=$ & \\
\hline Raphidioptera & Raphidiidae & Raphidia sp. & \\
\hline \multirow[t]{4}{*}{ Coleoptera } & Curculionidae & $=$ & \\
\hline & Staphylinidae & $=$ & $=$ \\
\hline & Carabidae & $=$ & \\
\hline & Dytiscidae? & $=$ & \\
\hline \multirow[t]{6}{*}{ Hymenoptera } & Ichneumonidae & $=$ & \\
\hline & Chalcidoidea & $=$ & \\
\hline & Apidae & Apis sp. & $=$ \\
\hline & Formicidae & Lasius? sp., Myrmicinae & \\
\hline & Diapriidae & $=$ & $=$ \\
\hline & Braconidae & $=$ & \\
\hline Trichoptera & Familia indet. & $=$ & \\
\hline Lepidoptera & Zygaenidae & Zygaena? turolensis & \\
\hline \multirow[t]{7}{*}{ Diptera } & Bibionidae & Bibio sp., Plecia sp. & $=$ \\
\hline & Mycetophilidae & Mycetophilinae & Mycetophilinae \\
\hline & Tipulidae & Dicranomyia sp., Tipula sp. & \\
\hline & Empididae & Hilara royoi, Hilara sp. & \\
\hline & Chironomidae & 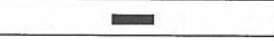 & \\
\hline & Culicidae & $=$ & \\
\hline & Otitidae & Seioptera? sp. & \\
\hline
\end{tabular}

Tabla 2. Listas faunísticas de los insectos de Río Rubielos (RR) y de Alto de la Venta (AV). Los taxones en negrita corresponden a nuevas citas para la cuenca. El símbolo $=$ indica únicamente presencia de la familia.

\section{Alto de la Venta (AV)}

El paraje de Alto de la Venta, al oeste de la población, presenta también un gran número de afloramientos con ritmitas bituminosas. Aquí se ha muestreado un yacimiento rico en restos de vegetales y artrópodos, del cual proviene el único ejemplar de hoja roída por oruga hallado en la cuenca (Lám. 2, Fig. 11). Además, hemos recolectado restos de insectos en otros tres afloramientos, que han proporcionado, en conjunto, los taxa que se presentan en el cuadro 2.

Además de insectos, también se han recuperado en estos afloramientos restos de crustáceos, tanto ostrácodos como cladóceros del género Daphnia, así como ejemplares de Araneae.

Los restos fósiles procedentes de estos afloramientos se encuentran depositados en el Museo Paleontológico Municipal de Valencia (MPV).

\section{AGRADECIMIENTOS}

Queremos expresar nuestro agradecimiento a E. Barrón, R. Daams y T. Fernández-Marrón, del Dpto. de Paleontología de la Universidad Complutense de Madrid, por la ayuda prestada en la elaboración de este trabajo. Jordi Guillem, Roser Momparler, Esteban José Sánchez y José María Tortajada participaron en las campañas de excavación. Asimismo, agradecemos al Sr. Federico Górriz el permitirnos consultar su colección particular, y al Sr. Federico Alegre la autorización para realizar excavaciones en un terreno de su propiedad. También agradecemos a los revisores las sugerencias y correcciones realizadas.

Este trabajo ha sido posible, en parte, gracias a una subvención otorgada en 1992 por la Diputación General de Aragón, para realizar la excavación de Alto de Ballester 1, y también a una Ayuda a la Investigación concedida por el Instituto de Estudios Turolenses en 1993, para abordar una excavación de las ritmitas bituminosas de Río Rubielos.

\section{BIBLIOGRAFÍA}

Aguirre, E. et Moissenet, E. 1972. Précisions sur le gisement miocène de Rubielos de Mora (Province de Teruel, Espagne). Melanges de la Casa de Velázquez, 8, 561-564.

Álvarez-Ramis, C. y Fernández-Marrón, T. 1994. Conexiones establecidas entre los palinomorfos y los macrorestos vegetales del Mioceno medio de Rubielos de Mora (Teruel). In: Polen y esporas: contribución a su conocimiento. VIII Simposio de Palinología (A.P.L.E.) (Ed. Irene La-Serna), Tenerife, 323-331.

Álvarez Sierra, M. A. and Daams, R. 1987. Pseudotheridomys fejfa$r i$, a new species of Eomyidae (Rodentia) from the Lower Miocene of North Teruel (Spain). Scripta Geologica, 83, 19-26.

Anadón, P. 1983. Características generales de diversas cuencas lacustres terciarias con pizarras bituminosas del NE de la Península Ibérica. Comunicaciones del X Congreso Nacional de Sedimentología, Menorca, 1, 9-12.

Anadón, P., Cabrera, L., Inglés, M., Julià, R. and Marzo, M. 1988a. The Miocene lacustrine basin of Rubielos de Mora, Excursion Guidebook, International workshop-field seminar on lacustrine facies models in rift systems and related natural resources, 1-32. Barcelona-Rubielos de Mora.

Anadón, P., Cabrera, L. and Julià, R. 1988b. Anoxic-oxic cyclical lacustrine sedimentation in the Miocene Rubielos de Mora Basin, Spain. In: Lacustrine Petroleum Source Rocks. (Eds. A.J.Fleet, K.Kelts \& M.R. Talbot). Geological Society London Special Publication, 40, 353-367.

Anadón, P., Cabrera, L., Julià, R., Roca, E. and Rosell, L. 1989. Lacustrine oil-shale basins in tertiary grabens from NE Spain (western european rift system). Palaeogeography, Palaeoclimatology, Palaeoecology, 70, 7-28

Anadón, P.; Moissenet, E. and Simón, J. L. 1990. The neogene grabens of the eastern iberian chain (eastern Spain). Paleontologia i Evolució, Memòria Especial, 2, 99-130.

Antunes, M. T., Azanza, B., Morales, J. y Soria, D. 1994. Los rumiantes (Artiodactyla, Mammalia) del Mioceno inferior del área de Lisboa (Cuenca del Tajo, Portugal). Revista Española de Paleontología, 9, 51-71.

Azanza, B. 1993. Systématique et évolution du genre Procervulus, cervidé (Artiodactyla, Mammalia) du Miocène inférieur d'Eu- 
rope. Comptes Rendus de la Academie des Sciences de Paris, 316, 717-723.

Azanza, B., Cerdeño, E., Ginsburg, L., Made, J. v.d., Morales, J. et Tassy, P. 1993. Les grands mammifères du Miocène inférieur d'Artesilla, bassin de Calatayud-Teruel (province de Saragosse, Espagne). Bulletin Muséum national Histoire natural. Paris, $4^{\mathrm{e}}$ sér., 15, sec. C, n. 1-4, 105-153.

Belinchón, M. 1987. Estudio tafonómico y sistemático de la fauna de mamíferos del Mioceno de Buñol (Valencia). Tesis doctoral, Univ. de València, 1-433.

Belinchón, M. y Montoya, P. 1989-1990. Presencia de Phyllotillon naricus Pilgrim, 1910 (Chalicotheriidae, Perissodactyla, Mammalia) en el Aragoniense de Buñol (Valencia). Breve síntesis de los calicotéridos en el registro español. Paleontologia i Evolució, 23, 171-180.

Bruijn, H. de and Moltzer, J. G. 1974. The rodents from Rubielos de Mora: the first evidence of the existence of different biotopes in the Early Miocene of eastern Spain. Proceedings Koninkle Nederlanden Akademie Van Wetenschappen, B, 77, 129-145.

Bruijn, H. de, Daams, R., Daxner-Höck, G., Fahlbusch, V., Ginsburg, L., Mein, P. and Morales, J. 1992. Report of the RCMNS working group on fossil mammals, Reisensburg 1990. Newsletter Stratigraphy, 26 (2/3): 65-118.

Cerdeño, E. 1989. Revisión de la sistemática de los rinocerontes del Neógeno de España. Tesis doctoral, Univ. Complutense de Madrid, $429 \mathrm{pp}$.

Chica, T. y Peñalver, E. 1990. Yacimiento paleoherpetológico de Rubielos de Mora (Teruel). Resumos I Congresso Luso-Espanhol (V Congresso Espanhol) de Herpetología, Lisboa, p 22.

Coombs, M.C. 1978. Reevaluation of Early Miocene north american Moropus (Perissodactyla, Chalicotheriidae, Schizotheriinae). Bulletin Carnegie Museum Natural History, 4, 1-62.

Coombs, M.C. 1989. Interrelationships and diversity in the Chalicotheriidae. In: The Evolution of Perissodactyls, (Eds. D.R. Prothero \& R.M. Schoch), Oxford Univ. Press, 438-457.

Crusafont, M., Gautier, F. et Ginsburg, L. 1966. Mise en évidence du Vindobonien inférieur continental dans l'Est de la provin- ce de Teruel (Espagne). Compte Rendu Sommaire des Séances de la Société Géologique de France, 1966 (1), 30-31.

Daams, R. and Freudenthal, M. 1990. The Ramblian and the Aragonian: limits, subdivision, geographical and temporal extension. In: European Neogene Mammal Chronology edited by E. H. Lindsay et al. Plenum Press, New York. Reisensburg, 1-658.

Daams, R., Freudenthal, M., and Alvarez Sierra, M. A. 1987. Ramblian; a new stage for continental deposits of early Miocene age. Geologie en Mijnbouw, 65, 297-308.

Fernández-Marrón, T. et Alvarez-Ramis, C. 1988. Note preliminaire sur l'étude paléobotanique du gisement de Rubielos de Mora (Teruel, Espagne). Résumés Seminaire de Paleobotanique. Organisation Francaise de Paleobotanique, Lille, p 7.

Fernández-Navarro, L. 1914. La cuenca petrolífera de Rubielos de Mora. Revista de la Academia de Ciencias, 13, 237-255.

Fernández Rubio, F. y Peñalver, E. 1994. Un nuevo ejemplar fósil de Zygaena ? turolensis Fernández-Rubio, Peñalver y Martínez-Delclós, 1991 (Lepidoptera, Zygaenidae). Estudios del Museo de Ciencias Naturales de Álava, 9, 39-48.

Fernández-Rubio, F.; Peñalver, E. y Martínez-Delclòs, X. 1991. Zygaena? turolensis, una nueva especie de Lepidoptera Zygaenidae del Mioceno de Rubielos de Mora (Teruel). Descripción y filogenia. Estudios del Museo de Ciencias Naturales de Alava, 6, 77-93.

Gavala, J. 1921. Nota acerca de los yacimientos de lignitos y pizarras bituminosas de Rubielos de Mora (Teruel). Bol. I.G.M.E., XLII, $2^{\mathrm{a}}$ ser., 263-302.

Gibert, J. 1974. Étude des insectivores du Miocène du Vallès-Penedès, Calatayud-Daroca et Rubielos de Mora. Thèse, Sabadell.

Gibert, J. 1975. New insectivores from the Miocene of Spain. Proceedings Koninkl. Nederl. Akademie Van Wetenschappen, B, 78 (2), 108-133.

Ginsburg, L. et Bulot, C. 1987. Les Artiodactyles sélénodontes du Miocène de Bézian à La Romieu (Gers). Bulletin Muséum national Histoire natural. Paris , 4e sér., 9, C (1), 63-95.

Godoy, A. y Anadón, P. 1986. Memoria y Mapa Geológico de España E. 1:50.000, $2^{\mathrm{a}}$ ser. Hoja 591. Mora de Rubielos. Instituto Geológico y Minero de España, 1-52.

Lámina I: macromamíferos de Alto de Ballester 1. MPZ: Museo Paleontológico de la Universidad de Zaragoza; MPV: Museo Paleontológico Municipal de Valencia; CFA: Colección Federico Alegre. Escalas $=5 \mathrm{~cm}$. 1: fragmento de mandíbula izquierda con $\mathrm{M}_{1-2}$ de Procervulus dichotomus (MPZ AB-49). 2: $\mathrm{M}_{3}$ derecho de Procervulus dichotomus (MPZ AB-24). 3: $\mathrm{M}^{3}$ derecho de Anchitherium sp. (MPZ AB-33). 4: $\mathrm{P}^{1}-\mathrm{P}^{3}$ derechos de Anchitherium sp. (CFA AB-101). 5: decidual inferior de cf. Brachypotherium sp. (MPV AB-4). 6: metatarsiano III izquierdo de Moropus sp. (MPV AB-13). 7: metatarsiano IV izquierdo de Moropus sp. (MPV AB-12). 8: dúplex de Moropus sp. (MPV AB-10). a, vista dorsal; b, vista medial. 9: tercera falange de Moropus sp. (MPV AB-18). a, vista lateral; b, vista dorsal.

Lámina II: asociación fósil en las ritmitas bituminosas de Río Rubielos, Alto de la Venta y Cerro del Porpol. Ejemplares depositados en el Museo Paleontológico de Valencia (MPV). 1: hoja de Zelkova zelkovaefolia (E. Barrón, com. pers.), de la familia Ulmaceae, de Río Rubielos (MPV-739-RM). Escala = 2 mm. 2: plumón de ave de Río Rubielos (MPV-1656-RM). Escala $=2 \mathrm{~mm}$. 3: estado juvenil de anfibio urodelo posiblemente del género Chelotriton de Río Rubielos (MPV-1655-RM). Escala $=5 \mathrm{~cm}$. 4: ortóptero Caelifera de Río Rubielos (MPV-246-RM). Escala $=5 \mathrm{~mm}$. 5: coleóptero de la familia Staphylinidae de Río Rubielos (MPV-260-RM). Escala = 2 mm. 6: himenóptero de la familia Apidae de Río Rubielos (MPV-270-RM). Escala $=2 \mathrm{~mm}$. 7: heteróptero de la familia Lygaeidae de Río Rubielos (MPV-311-RM). Escala = $5 \mathrm{~mm}$. 8: homóptero de la familia Aphididae de Río Rubielos (MPV-261-RM). Escala = $1 \mathrm{~mm}$. 9: díptero de la familia Otitidae de Río Rubielos (MPV30-RM). Escala = $1 \mathrm{~mm}$. 10: díptero tipúlido del género Dicranomyia de Río Rubielos (MPV-805-RM). Escala = $2 \mathrm{~mm} .11$ : hoja con mordeduras de oruga de Alto de la Venta (MPV-1025-RM). Escala = $5 \mathrm{~mm}$. 12: homóptero de la familia Psyllidae de Cerro del Porpol (MPV-1654-RM). Escala = $1 \mathrm{~mm}$. 13: dos ejemplares de tisanópteros de la familia Thrypidae con detalle aumentado de uno de ellos (MPV-1650-RM y MPV-1651-RM) de Cerro del Porpol. Escalas = $1 \mathrm{~mm}$. 14: himenóptero de la familia Ichneumonidae de Cerro del Porpol (MPV-1652-RM). Escala = $1 \mathrm{~mm}$. 15: coleóptero de la familia Curculionidae de Cerro del Porpol (MPV-1653-RM). Escala = $1 \mathrm{~mm}$. 
Lámina I
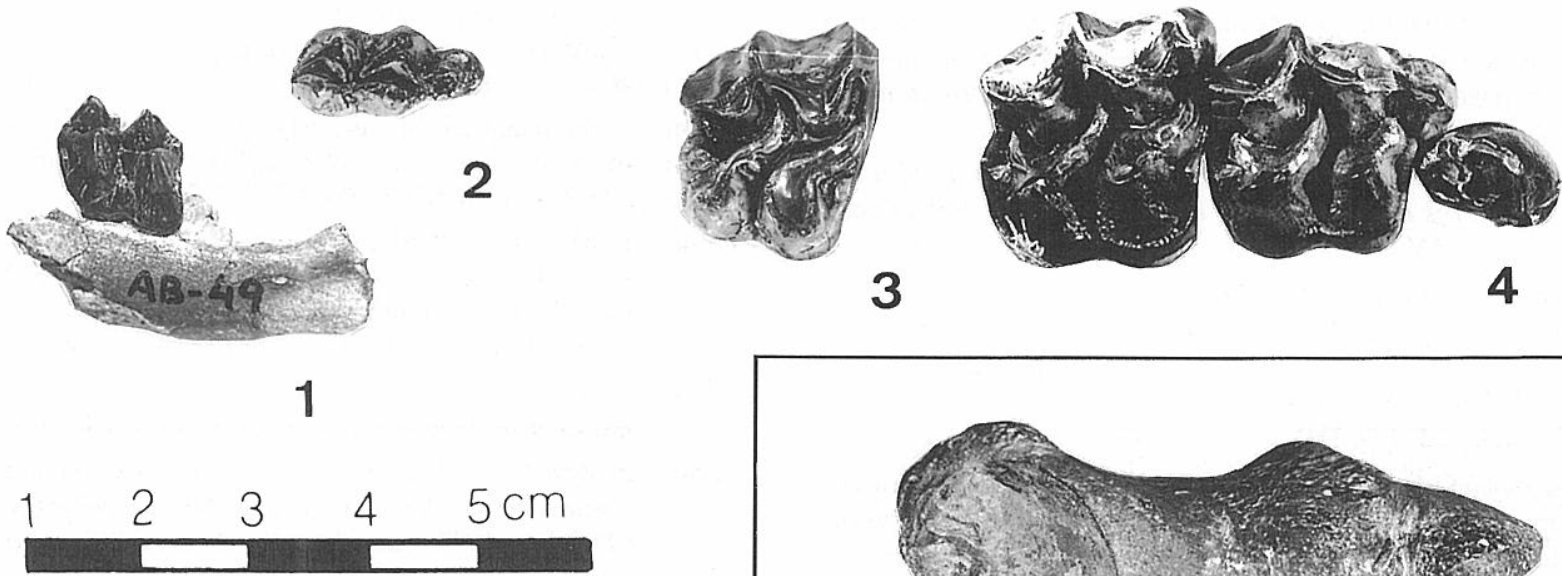

3

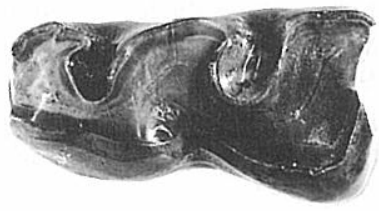

5

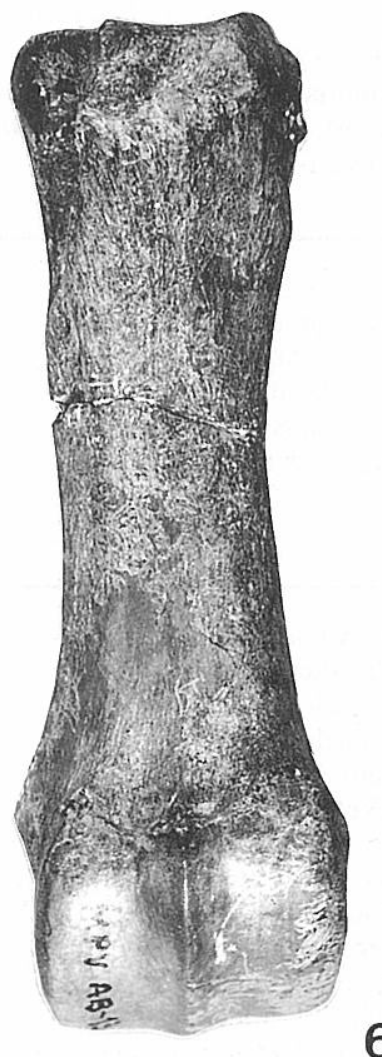

6

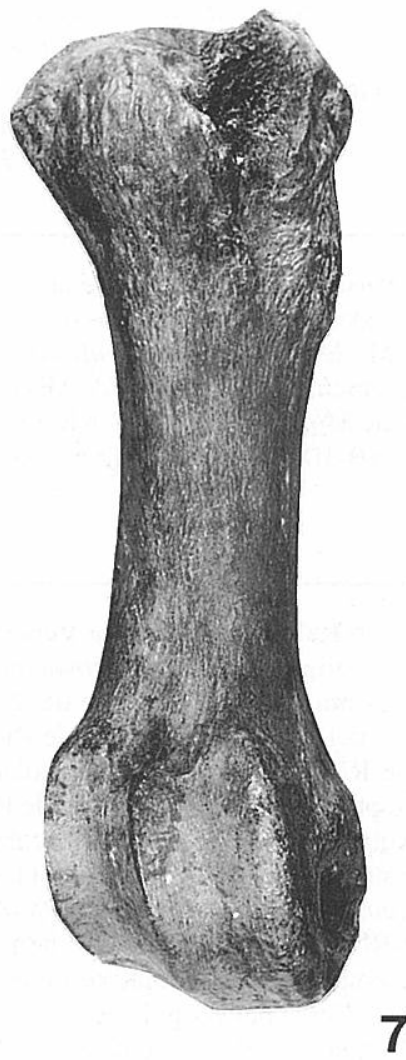

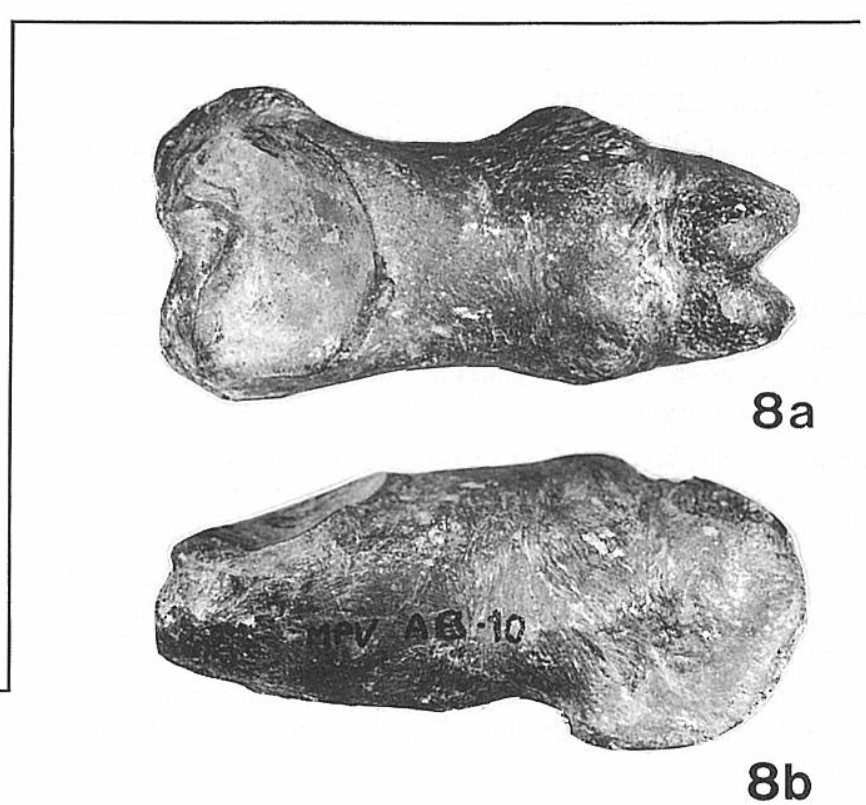
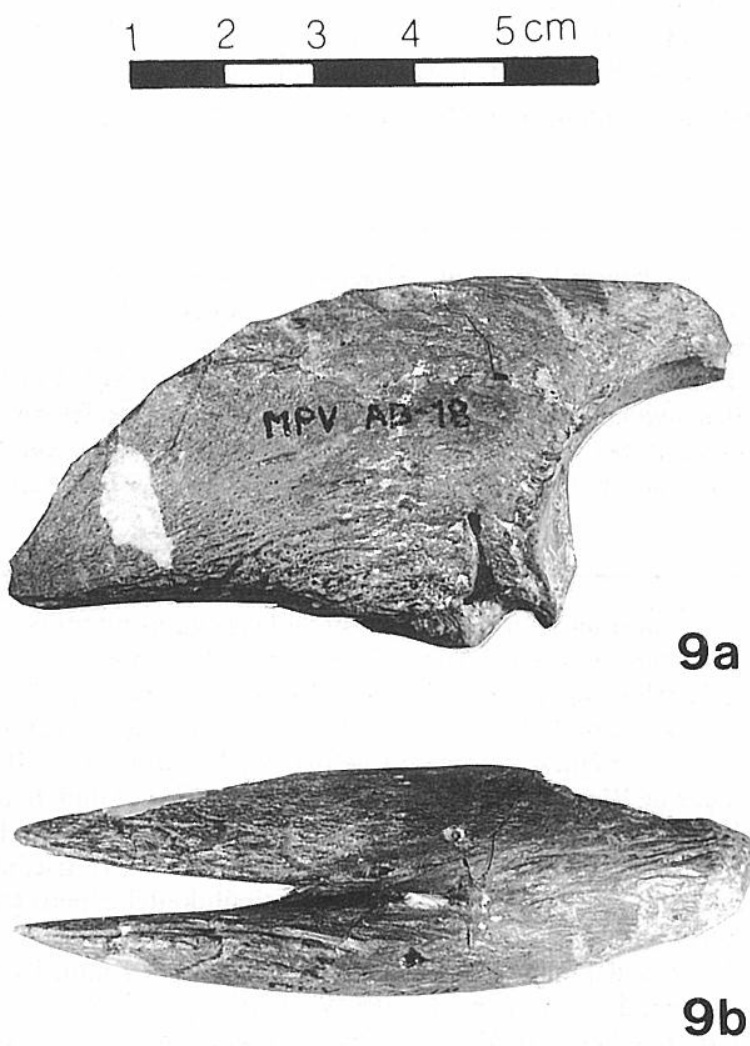
Lámina II
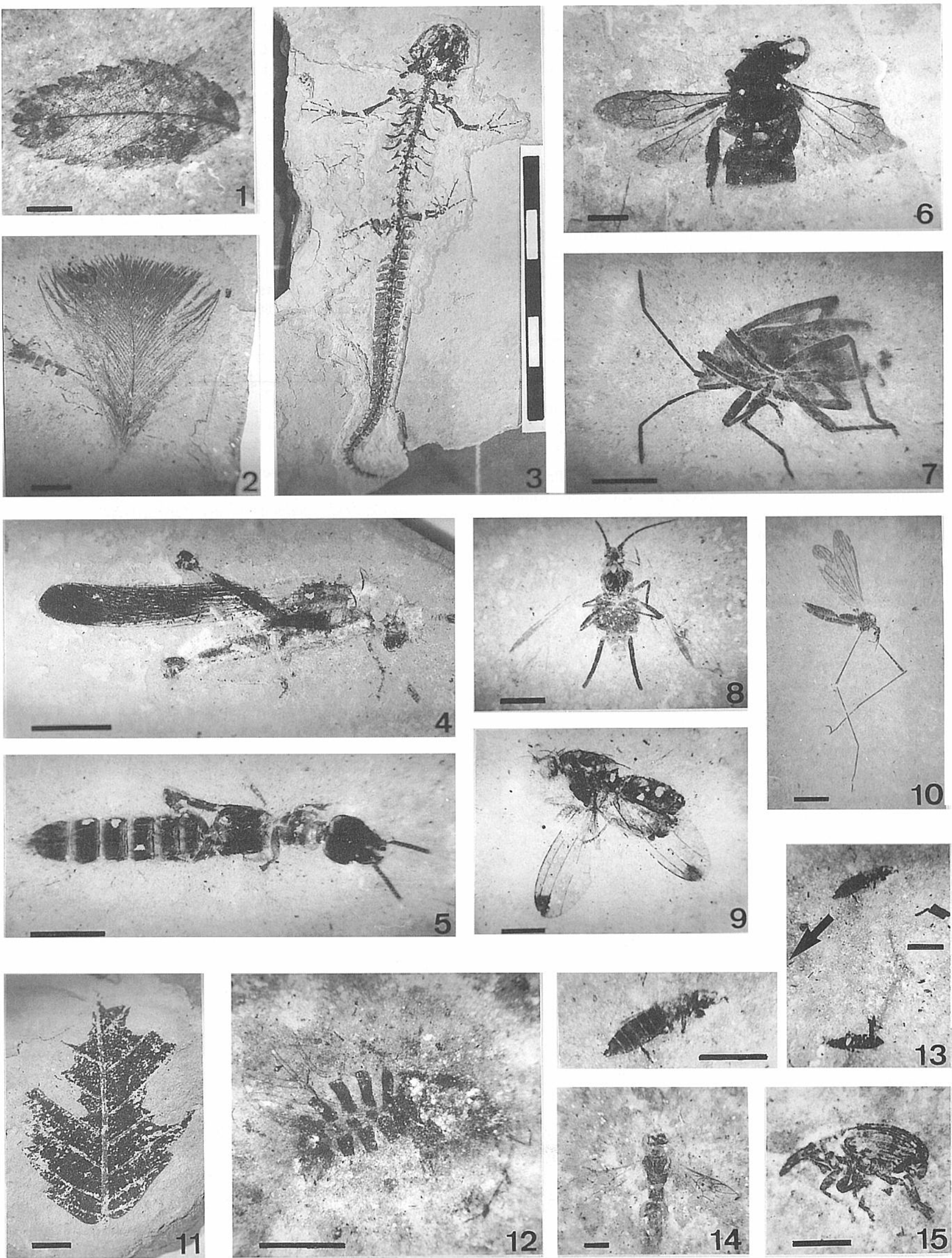
Guimerà, J. 1990. Formación de una cubeta sinclinal en un contexto extensivo: la cuenca miocena de Rubielos de Mora (Teruel). Geogaceta, 8, 33-35.

Hernández-Sampelayo, P. y Cincúnegui, M. 1926. Cuenca de esquistos bituminosos de Ribesalbes (Castellón). Boletín del Instituto Geológico y Minero de España, 6, 3ª ser., 3-86.

Herráez, E. y Alberdi, M. T. 1983. Anchitherium aurelianense del yacimiento del Puente de Vallecas. Estudios geológicos, 39, 409-415.

Íñigo, C. 1993. Estudio de los perisodáctilos del yacimiento mioceno de Córcoles (Guadalajara). Tesis doctoral inédita, Universidad Complutense de Madrid.

López-Martínez, N. 1977. Revisión sistemática y biostratigráfica de los Lagomorpha (Mammalia) del Terciario y Cuaternario de España. Tesis doctoral, Universidad Complutense, Madrid, 1-469.

López-Martínez, N. 1989. Revisión sistemática y biostratigráfica de los Lagomorpha (Mammalia) del Terciario y Cuaternario de España. Memorias del Museo Paleontológico de la Universidad de Zaragoza, 3, 1-350.

Martínez-Delclòs, X., Belinchón, M. y Peñalver, E. 1989. Insectos del Mioceno. Rubielos de Mora (Teruel). Resúmenes de comunicaciones V Jornadas de Paleontología, Valencia, 97-98.

Martínez-Delclòs, X., Peñalver, E. y Belinchón, M. 1991. Primeras aportaciones al estudio de los insectos del Mioceno de Rubielos de Mora, Teruel (España). Revista Española de Paleontología, nº Extraordinario, 125-137.

Mein, P. 1990. Updating of MN zones. In: European Neogene Mammal Chronology (Eds. E.H. Lindsay et al.). Plenum Press, New York, 73-90.
Moissenet, E. 1989. Les fossés néogènes de la chaine Ibérique: Leur évolution dans le temps. Bulletin de la Société Géologique de France, 5, 916-926.

Moissenet, E. et Gautier, F. 1971. La région de Rubielos de Mora (Province de Teruel, Chaînes Ibériques orientales). Contribution à l'étude géologique et morphologique. Mélanges de la Casa de Vélazquez, 7, 5-28.

Naumann, C. M. 1987. On the phylogenetic significance of two Miocene zygaenid moths (Insecta, Lepidoptera). Paläontologische Zeitschrifft, 61, 299-308.

Peñalver, E. and Martínez-Delclòs, X. 1993. Taphonomical aspects of the fossil arthropods from Rubielos de Mora, Teruel (Spain). Premier Congrès Européen de Paléontologie, Lyon, p 100.

Prado, J. G., Suárez-Ruiz, I., Borrego, M. A. G. and García, A. M. 1988. The nature of organic matter in oil shales from Rubielos de Mora. Intern. Workshop "Lacustrine facies models in rift system and related natural resources" Barcelona-Rubielos de Mora. Abstracts, p 1.

Reyes, J. L. y Feixas, J. C. 1984. Las pizarras bituminosas: definición, composición y clasificación. I Congreso Español de Geología, II, 817-827.

Sanchiz, F. B. 1977. Catálogo de los anfibios fósiles de España (Noviembre de 1977). Acta Geológica Hispánica, 12, 103-107.

Simón, J. L. 1984. Compresión y distensión alpinas en la Cadena Ibérica oriental. Instituto de Estudios Turolenses de la Excelentísima Diputación de Teruel, Teruel, 1-269.

Manuscrito recibido: 30 de noviembre, 1994 Manuscrito aceptado: 19 de julio, 1995 